\title{
Multiwavelength observations of the Be star/X-ray binary EXO2030+375 during outburst
}

\author{
A.J. Norton, ${ }^{1}$ D. Chakrabarty, ${ }^{2}$ M.J. Coe,${ }^{3}$ C. Everall, ${ }^{3}$ M.H. Finger, ${ }^{4}$ \\ T.A. Prince, ${ }^{2}$ P. Roche, ${ }^{3}$ M.T. Stollberg ${ }^{5}$ and R.B. Wilson ${ }^{4}$ \\ ${ }^{1}$ Department of Physics, The Open University, Walton Hall, Milton Keynes MK7 6AA \\ ${ }^{2}$ Palomar Observatory and Space Radiation Laboratory 220-47, California Institute of Technology, Pasadena, California 91125, USA \\ ${ }^{3}$ Astronomy and Space Physics Group, Department of Physics, University of Southampton, Southampton SO9 5NH \\ ${ }^{4}$ Space Science Laboratory, NASA Marshall Space Flight Center, Huntsville, Alabama 35812, USA \\ ${ }^{5}$ Department of Physics, University of Alabama, Huntsville, Alabama 35809, USA
}

Accepted 1994 August 17. Received 1994 August 15; in original form 1994 March 2

\begin{abstract}
We present the results from a campaign to monitor the behaviour of the Be star/Xray binary EXO2030+375 during an X-ray outburst. The BATSE all-sky monitor on board the Compton Gamma-Ray Observatory satellite regularly detects pulsed emission from EXO2030+375 when the neutron star is near periastron. Ground-based optical spectroscopic data plus infrared photometric and polarimetric data were obtained simultaneously with these X-ray data during a typical outburst in 1993 June/July. Despite an increase in the pulsed X-ray flux by an order of magnitude, neither the infrared luminosity nor the equivalent width, strength or profile of the $\mathrm{H} \alpha$ emission line showed any significant, correlated changes. We conclude that (i) reprocessing of the X-ray flux in the Be star circumstellar envelope, (ii) gravitational disruption of that envelope by the neutron star and (iii) enhanced emission from an HiI region formed around the neutron star are all minimal effects and below our detection threshold. We have also detected significant linear polarization in the infrared flux from EXO2030+375, and we discuss whether or not some fraction of this is intrinsic to the source.
\end{abstract}

Key words: binaries: close - circumstellar matter - stars: individual: EXO2030+375 stars: neutron - infrared: stars - X-rays: stars.

\section{INTRODUCTION}

The EXO2030+375 system consists of a neutron star in an eccentric 46-d orbit around a 20th-magnitude Be star companion (Motch \& Janot-Pacheco 1987; Coe et al. 1988; Janot-Pacheco, Motch \& Pakull 1988). In common with Be stars in general, the primary is thought to be surrounded by an envelope of material which is responsible for the characteristic infrared excess and Balmer emission lines that are seen (e.g. Slettebak 1979). At periastron, the neutron star passes through the densest part of this circumstellar material, giving rise to enhanced accretion on to the neutron star surface. As a result of this, the X-ray emission (pulsed at the neutron star spin period of $41.8 \mathrm{~s}$ ) increases dramatically (Parmar et al. 1989a; Parmar, White \& Stella 1989b; Stollberg et al. 1993; Reynolds, Parmar \& White 1993). This produces the transient, outburst behaviour that is commonly seen in Be star/X-ray binaries (e.g. White, Swank \& Holt 1983; van den Heuvel \& Rappaport 1987; Nagase 1989; Waters 1989), in this case periodically at the binary orbital period.
To investigate this behaviour, we organized a multiwavelength observational campaign during a predicted X-ray outburst in 1993 June/July. Using a combination of optical spectroscopy, infrared photometry and polarimetry, plus continuous X-ray data, the aim of this campaign was to investigate the effect that the passage of the neutron star has on the circumstellar material expelled by the Be star. The X-ray emission is a direct probe of conditions existing near the neutron star, whilst the $\mathrm{H} \alpha$ emission line and the infrared continuum are directly related to conditions in the inner circumstellar envelope through which the neutron star passes at periastron. As we were able to predict when the X-ray outburst would occur, we were able to obtain simultaneous optical and infrared measurements in conjunction with X-ray data throughout the outburst. This is one of the first times that such a campaign has been possible for a system of this type. A multiwavelength campaign to observe the Be star/X-ray binary A1118-616 has also recently been carried out, as reported by Coe et al. (1994). In that case, optical, infrared and ultraviolet observations of A1118-616 were made soon after the onset of a 
large (unpredicted) X-ray outburst detected by BATSE. We therefore compare our results from EXO2030+375 with those from A1118-616 and other Be star/X-ray binaries. As will be seen, there are significant differences between the behaviours of these two, recently well-studied, systems.

\section{OBSERVATIONS}

\subsection{Compton Gamma-Ray Observatory}

The Burst and Transient Source Experiment (BATSE) is an all-sky monitor instrument on board the Compton Gamma-Ray Observatory (CGRO) satellite (see Fishman et al. 1989 for a description of BATSE). Using its eight detectors mounted on the corners of the spacecraft, BATSE detects many X-ray transients and pulsars using both pulsed and occultation analyses of its continuous data (see Prince et al. 1994 for a review of BATSE observations of accreting pulsars). Since the beginning of its operation in 1991 April, BATSE has regularly detected pulsed emission from EXO2030+375 (Stollberg et al. 1993, 1994), characterized by the 41.8 -s period previously discovered using EXOSAT (Parmar et al. 1989a,b). Moreover, this period, due to the neutron star spin, displays Doppler shifts that are evidence of binary motion around the Be star. From these data, improved orbital elements of the system have been derived, namely $P_{\text {orb }}=46.03 \pm 0.01 \mathrm{~d}, e=0.33 \pm 0.03, a_{\mathrm{x}} \sin i=268 \pm 25$ light seconds, $\omega=228.2 \pm 5.7$ and $T_{\text {peri }}=J D 2448798.7 \pm 0.3$ (Stollberg et al. 1994). In fact, BATSE detects pulsed emission from EXO2030+375 only in $\mathrm{a} \approx 2$-week interval that begins just after periastron, but does so on almost every binary orbit. The regularity of these outbursts enabled the scheduling of ground-based observations to coincide with the predicted outburst of 1993 June/July.

\subsection{UK Infrared Telescope}

Observations using the 3.8-m United Kingdom Infrared Telescope (UKIRT) at the Mauna Kea observatory, Hawaii, were carried out on the nights of 1993 June 28 - 30, and then again on 1993 July $2-6$. The first three and last two nights were observer led, whilst, for three nights in the middle of the run, service observations were obtained. The original aim of the campaign had been to obtain infrared spectroscopy using the Cooled Grating Spectrometer (CGS4). In the event, however, CGS4 was inoperative and the Infrared Camera (IRCAM) was used instead to provide $J$-, $H$-, $K$ - and $L^{\prime}$-band imaging photometry. IRCAM was used rather than the photometer, UKT9, because on several nights the conditions were not photometric throughout, but relative photometry could be performed with respect to another star in the IRCAM field. IRCAM was used in its 0.62 arcsec pixel ${ }^{-1}$ mode. Exposure times were $1 \mathrm{~s} \times 30$ co-adds (i.e. $30 \mathrm{~s}$ ) at $J, H$ and $K$ (repeated) and $0.2 \mathrm{~s} \times 50$ co-adds (i.e. $10 \mathrm{~s}$ ) at $L^{\prime}$. The nearby star HD203856 (BD+394542) was also observed when conditions were suitable in order that photometric flux calibration could be performed.

In addition to these photometric data obtained during the X-ray outburst, two additional sets of service data were obtained on 1993 June 22 (just before the X-ray outburst, at orbital phase 0.872) and on 1993 August 11 (some six weeks after the X-ray outburst, at orbital phase 0.953). These data were obtained using UKT9, in photometric conditions.

Interspersed with the photometric data, $J$ - and $K$-band polarimetric data were also obtained on the first three and last two nights of the campaign. Four images on each night were obtained, with the waveplate at $0^{\circ}, 45^{\circ}, 22^{\circ} .5$ and 67.5 . Exposure times were $2 \mathrm{~s} \times 30$ co-adds (i.e. $60 \mathrm{~s}$ ) in each case. The bipolar nebula S106 was observed as a polarimetric calibration source on two occasions: once during the first three nights of the campaign, and once during the last two nights. This calibration was sufficient since the waveplate position is likely to be reproducible to a fraction of a degree over a few-day period unless the waveplate is removed from the polarimeter.

\subsection{Hale telescope and William Herschel telescope}

Spectroscopic observations using the double spectrograph (Oke \& Gunn 1982) at the f/15.7 Cassegrain focus of the 5-m (200-inch) Hale telescope at Palomar Mountain, California (hereafter P200), were carried out on the nights of 1993 June $28-29$. Spectra were obtained at $2-\AA$ resolution in the blue arm and $6-\AA$ resolution in the red arm on both nights, and also at $0.8-\AA$ resolution in the red arm on the second night. Exposure times used were typically $600 \mathrm{~s}$. The nearby $\mathrm{B}$ star HD227900 (BD+40 4032) was observed as a spectrophotometric standard.

Spectroscopic observations using the ISIS double spectrograph (Clegg et al. 1992) at the f/11 Cassegrain focus of the 4.2-m William Herschel telescope (WHT) at the Roque de los Muchachos Observatory, La Palma, were carried out on the nights of 1993 June $29-30$. Spectra were obtained at $3-\AA$ and $0.7-\AA$ resolution in the red arm on both nights; at $3-\AA$ resolution in the blue arm on both nights; and at $0.8-\AA$ resolution in the blue arm on the second night. Exposure times of $1000 \mathrm{~s}$ were used in each case. HD227900 was again observed as a spectrophotometric standard.

In addition to this, a single spectrum of EXO2030+375 was obtained in service time on the night of 1993 June 24, just prior to the X-ray outburst. The red arm spectrum was obtained at the same $0.7-\AA$ resolution as later WHT spectra, but no spectrophotometric standard observations were made.

At both the WHT and Palomar, bias frames and tungsten lamp flats were taken at the beginning and end of each night's observing. Calibration arcs were taken typically after every two object spectra and always after any telescope movements to enable correction to be made for flexure of the instrument. A summary of the spectra obtained is given in Table 1, showing the orbital phase of EXO2030+375 corresponding to each observation. Note that phase zero corresponds to periastron in this and subsequent tables.

\section{DATA REDUCTION AND RESULTS}

\subsection{Hard X-ray data}

BATSE observations of EXO2030+375 spanned the whole outburst, from a first detection of pulsed X-ray emission on 1993 June 28 (orbital phase 0.01) through to the last detection on 1993 July 8 (orbital phase $\sim 0.24$ ). Note that, in Figs 1, 2 and 3 below, we show only the pulsed X-ray flux from EXO2030+375. This is all that can be determined, since 
Table 1. Observation log for optical spectra.

\begin{tabular}{|c|c|c|c|c|}
\hline Telescope & $\begin{array}{l}\text { JD } \\
2440000+\end{array}$ & $\begin{array}{l}\text { Orbital } \\
\text { Phase }\end{array}$ & $\begin{array}{l}\text { Disp. } \\
(\AA / \text { pix })\end{array}$ & $\begin{array}{l}\text { Spectral } \\
\text { Range }(\AA)\end{array}$ \\
\hline WHT & 9163.703 & 0.930 & 1.54 & $3500-5100$ \\
\hline WHT & 9163.703 & 0.930 & 0.73 & $6300-7200$ \\
\hline P200 & 9167.913 & 0.021 & 2.1 & $3500-5200$ \\
\hline P200 & 9167.913 & 0.021 & 6.1 & $5100-10000$ \\
\hline P200 & 9167.925 & 0.021 & 6.1 & $5100-10000$ \\
\hline P200 & 9167.931 & 0.022 & 6.1 & $5100-10000$ \\
\hline WHT & 9168.446 & 0.033 & 2.88 & $3300-6300$ \\
\hline WHT & 9168.446 & 0.033 & 2.72 & $5700-9100$ \\
\hline WHT & 9168.463 & 0.033 & 2.88 & $3300-6300$ \\
\hline WHT & 9168.463 & 0.033 & 2.72 & $5700-9100$ \\
\hline WHT & 9168.482 & 0.034 & 2.72 & $5700-9100$ \\
\hline WHT & 9168.536 & 0.035 & 0.73 & $6150-7050$ \\
\hline WHT & 9168.549 & 0.035 & 0.73 & $6150-7050$ \\
\hline WHT & 9168.564 & 0.036 & 0.73 & $6150-7050$ \\
\hline WHT & 9168.578 & 0.036 & 0.73 & $6150-7050$ \\
\hline WHT & 9168.591 & 0.036 & 0.73 & $6150-7050$ \\
\hline WHT & 9168.607 & 0.037 & 0.73 & $6150-7050$ \\
\hline WHT & 9168.621 & 0.037 & 0.73 & $6150-7050$ \\
\hline WHT & 9168.636 & 0.037 & 0.73 & $6150-7050$ \\
\hline P200 & 9168.931 & 0.043 & 2.1 & $3500-5200$ \\
\hline P200 & 9168.931 & 0.043 & 6.1 & $5100-10000$ \\
\hline P200 & 9168.939 & 0.043 & 2.1 & $3500-5200$ \\
\hline P200 & 9168.939 & 0.043 & 6.1 & $5100-10000$ \\
\hline P200 & 9168.949 & 0.044 & 2.1 & $3500-5200$ \\
\hline P200 & 9168.949 & 0.044 & 6.1 & $5100-10000$ \\
\hline P200 & 9168.961 & 0.044 & 2.1 & $3500-5200$ \\
\hline P200 & 9168.961 & 0.044 & 6.1 & $5100-10000$ \\
\hline P200 & 9169.007 & 0.045 & 2.1 & $3500-5200$ \\
\hline P200 & 9169.007 & 0.045 & 0.82 & $6150-6800$ \\
\hline P200 & 9169.012 & 0.045 & 2.1 & $3500-5200$ \\
\hline P200 & 9169.012 & 0.045 & 0.82 & $6150-6800$ \\
\hline P200 & 9169.017 & 0.045 & 2.1 & $3500-5200$ \\
\hline P200 & 9169.017 & 0.045 & 0.82 & $6150-6800$ \\
\hline P200 & 9169.022 & 0.045 & 2.1 & $3500-5200$ \\
\hline P200 & 9169.022 & 0.045 & 0.82 & $6150-6800$ \\
\hline WHT & 9169.488 & 0.055 & 0.79 & $4400-5200$ \\
\hline WHT & 9169.488 & 0.055 & 0.73 & $6150-7050$ \\
\hline WHT & 9169.506 & 0.055 & 0.79 & $4400-5200$ \\
\hline WHT & 9169.506 & 0.055 & 0.73 & $6150-7050$ \\
\hline WHT & 9169.519 & 0.055 & 0.79 & $4400-5200$ \\
\hline WHT & 9169.519 & 0.055 & 0.73 & $6150-7050$ \\
\hline WHT & 9169.533 & 0.056 & 0.79 & $4400-5200$ \\
\hline WHT & 9169.533 & 0.056 & 0.73 & $6150-7050$ \\
\hline WHT & 9169.547 & 0.056 & 0.79 & $4400-5200$ \\
\hline WHT & 9169.547 & 0.056 & 0.73 & $6150-7050$ \\
\hline WHT & 9169.568 & 0.056 & 0.79 & $4400-5200$ \\
\hline WHT & 9169.568 & 0.056 & 0.73 & $6150-7050$ \\
\hline WHT & 9169.584 & 0.057 & 0.79 & $4400-5200$ \\
\hline WHT & 9169.584 & 0.057 & 0.73 & $6150-7050$ \\
\hline WHT & 9169.598 & 0.057 & 0.79 & $4400-5200$ \\
\hline WHT & 9169.598 & 0.057 & 0.73 & $6150-7050$ \\
\hline WHT & 9169.649 & 0.058 & 2.88 & $3300-6300$ \\
\hline WHT & 9169.649 & 0.058 & 2.72 & $5700-9100$ \\
\hline WHT & 9169.663 & 0.058 & 2.88 & $3300-6300$ \\
\hline WHT & 9169.663 & 0.058 & 2.72 & $5700-9100$ \\
\hline
\end{tabular}

the BATSE detectors are uncollimated and have wide fields of view. In normal operational mode the flux from many unresolved X-ray sources is therefore recorded as a single measurement. The X-ray flux pulsed at 41.8 -s is simply the pulsed component of the flux from EXO2030+375 which sits on top of a mean X-ray flux from that source plus all others in the field of view during each observation period. BATSE can

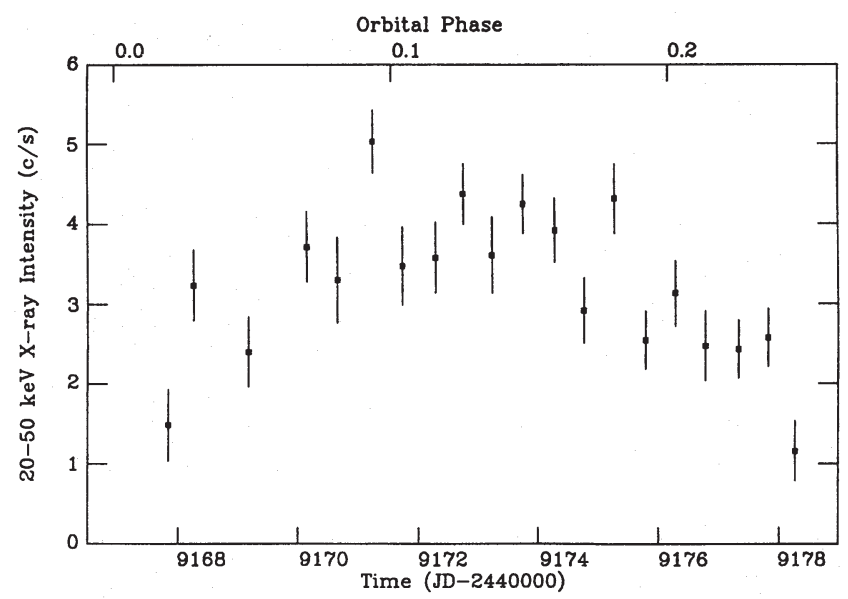

Figure 1. The BATSE X-ray intensity history of EXO2030+375 during the outburst. The intensity shown is the pulsed flux only, in the $20-50$ $\mathrm{keV}$ band.

only determine unpulsed flux levels by analysis of data near Earth occultations. During such observations, the disappearance of the 41.8-s pulsed component can be used to indicate what fraction of the total BATSE flux is due to EXO2030+375 alone.

\subsubsection{Light curve}

As can be seen from Fig. 1, the pulsed X-ray intensity of EXO2030+375 increased significantly over the course of about five days, followed by a decline over a similar time-scale. Prior to JD 2449167 and after JD 2449179 , the intensity of the pulsed X-ray emission from the source was below $\approx 0.5$ count $\mathrm{s}^{-1}$ in the $20-50 \mathrm{keV}$ band. This is the detection limit for BATSE and therefore EXO2030+375 is undetectable outside these times.

\subsubsection{Pulse profile}

Fig. 2 shows the mean pulse profile of EXO2030+375, averaged over the interval JD 244 9168-244 9178. The data shown in this figure are the result of subtracting a mean X-ray flux due to other X-ray sources in the BATSE field of view. Since this 'background' value is uncertain, the zero level shown in the pulse profile is also. Nothing should therefore be read into the 'negative count rate' shown in Fig. 2. What is real, however, is the peak-to-peak amplitude of $\sim 15$ count $s^{-1}$. This folded profile has been corrected for the variations in pulse period caused by orbital motion. There are hints that a double-peak structure may be present in the pulse profile, and this is consistent with that seen by EXOSAT during the 1985 outburst, at energies below $30 \mathrm{keV}$.

\subsubsection{Spectrum}

The mean X-ray spectrum obtained over the course of the outburst is shown in Fig. 3. It can be well fitted using a thermal bremsstrahlung model, with a temperature $k T=21.6 \pm 1.1 \mathrm{keV}$. The reduced chi-squared for this fit is 1.6 (6 degrees of freedom). Fits to the daily average X-ray spectra throughout the 


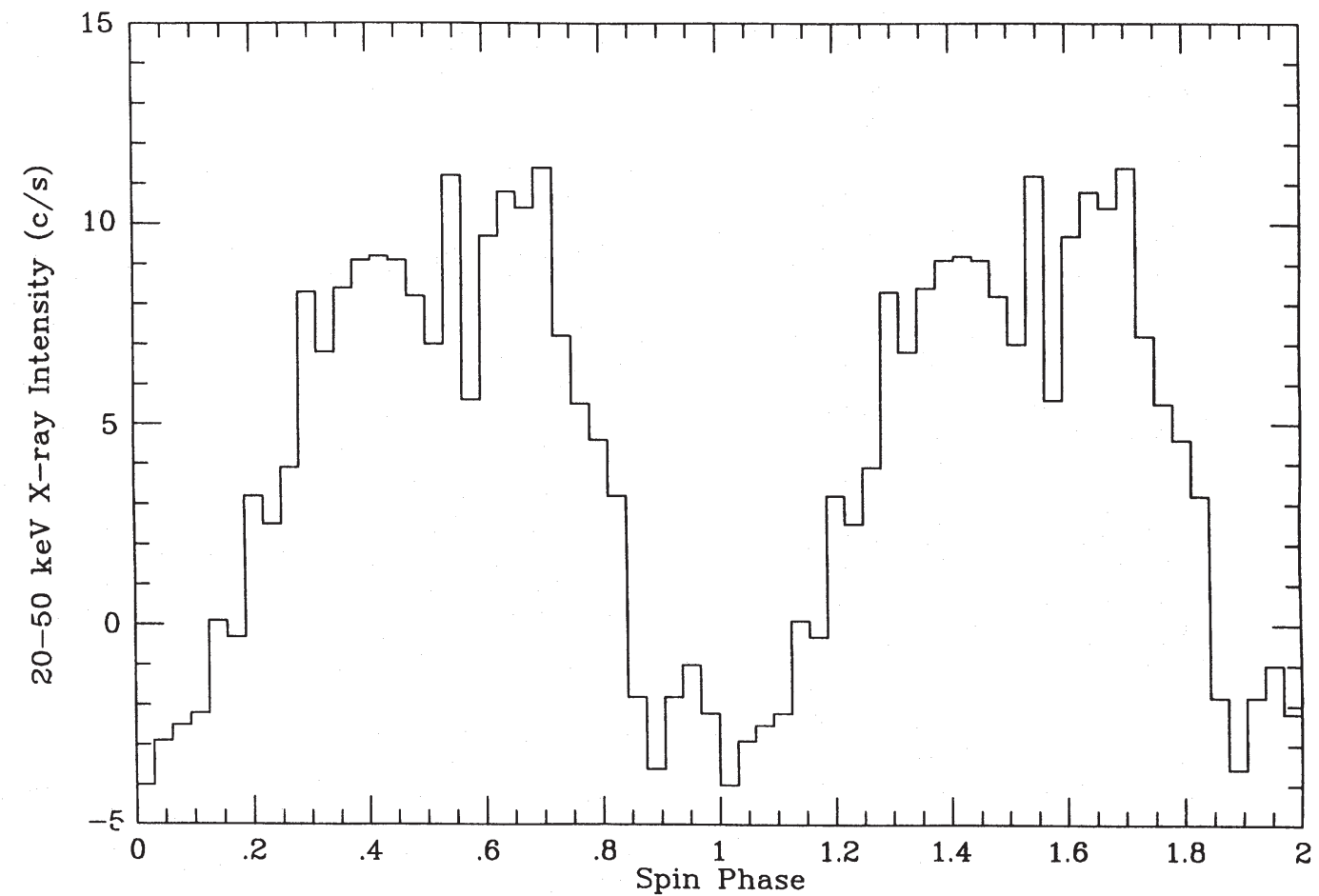

Figure 2. The mean BATSE X-ray pulse profile of EXO2030+375 during the outburst, repeated over two cycles for clarity. The intensity shown is the pulsed flux only, in the $20-50 \mathrm{keV}$ band. A typical error bar size is \pm 1 count $\mathrm{s}^{-1}$.

outburst yield temperatures in the range $\sim 15-40 \mathrm{keV}$; however, these parameters are in general not well constrained. A power-law model does not provide such a good fit to the mean spectrum (reduced chi-squared $=3.4$ ); however, daily X-ray spectra during the outburst are adequately fitted by power-law models. This may simply be due to the lower statistical quality of the daily spectra, when compared with the mean spectrum.

Extrapolation of the BATSE spectrum back to $\sim 1 \mathrm{keV}$ implies a total (unabsorbed) pulsed X-ray flux of $\sim(1.15 \pm$ $0.06) \times 10^{-9} \mathrm{erg} \mathrm{s}^{-1} \mathrm{~cm}^{-2}$. (The $1 \sigma$ confidence limits are based on the uncertainties in the spectral parameters only.) This may be compared with the $(1-20 \mathrm{keV}) \mathrm{X}$-ray flux observed by EXOSAT during the major outburst of 1985 , which reached a maximum of $\sim 3.3 \times 10^{-8} \mathrm{erg} \mathrm{s}^{-1} \mathrm{~cm}^{-2}$, close to the Eddington limit, assuming a distance of $5 \mathrm{kpc}$ and a neutron star mass of $1.44 \mathrm{M}_{\odot}$ (Parmar et al. 1989a).

\subsection{Infrared data}

\subsubsection{Photometry}

The infrared data from UKIRT were analysed using the Starlink IRCAM data reduction package (Aspin, Emerson \& Currie 1991). Images of the targets were dark-subtracted using observations made throughout each night, and then flat-fielded using an image of the same area taken immediately before or after, but shifted by 14 arcsec to the east or west. As the median was used to normalize the flat, the presence of a star had no effect. Bad pixels, of which there were about 40 on the $62 \times 58$ array, were removed by replacing with the average value of neighbouring pixels. Because EXO2030+375 was near the edge of the field and also because the field is relatively crowded, sky background from an annulus around the target could not be used. Instead, an $8 \times 8$ pixel area without any star (or star in the flat-field image) was used to subtract the average sky value of the image in each case.

A 10-arcsec aperture was used for the photometry, which was performed on the standard star, EXO2030+375 and the comparison star in the same field. As the standard zeropoints fluctuated, an average magnitude for the comparison star was calculated (ignoring obviously discrepant points), and the value for EXO2030+375 was adjusted accordingly. Errors were estimated from the fluctuations in the consecutive pairs of EXO2030+375 images and comparison star images. The observing conditions were not photometric for the whole of each night; conditions were particularly poor on the nights of June 29 and 30. Table 2 lists the total set of infrared photometric data obtained during the campaign, indicating the orbital phase of EXO2030+375 during each observation. Also shown are the two sets of service data obtained using UKT9, before and after the X-ray outburst.

The resulting four-colour infrared photometry, shown in Fig. 4, spans the whole of the X-ray outburst. This may be compared with Fig. 1 which shows the pulsed X-ray flux over the same interval. It is immediately apparent that, within the measurement uncertainty, the fluxes in all four infrared bands show no change that is correlated with the X-ray behaviour. Any systematic brightening or fading of the source at these wavelengths is less than one-tenth of a magnitude. We also note that the service data obtained on 1993 June 22 (prior to the outburst) are consistent with the data obtained during the outburst. However, this is not true of the service data obtained on 1993 August 11, after the outburst, which reveal EXO2030+375 to be in a fainter state. From these data alone 


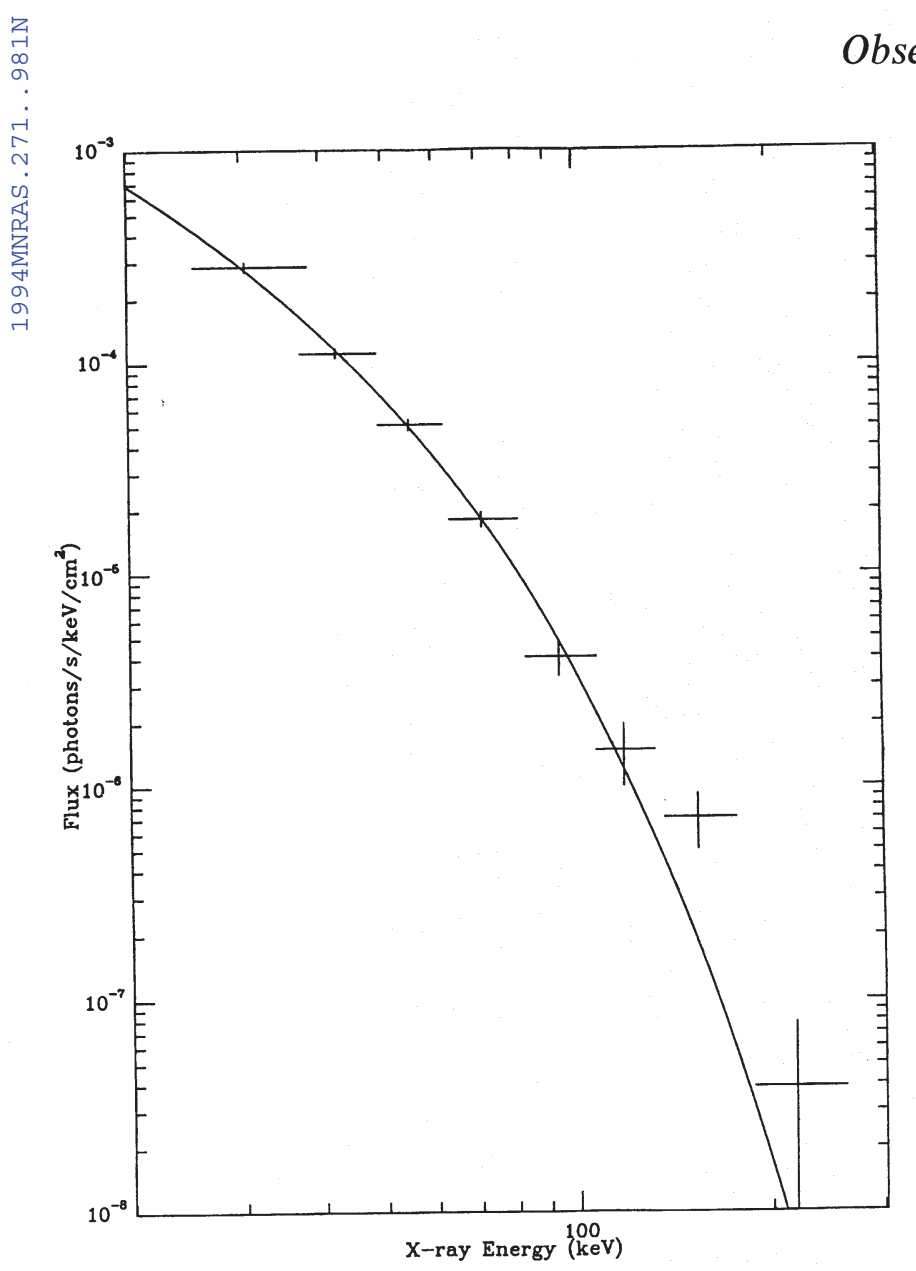

Figure 3. The mean BATSE X-ray spectrum of EXO2030+375 during the outburst. The solid line is the best-fitting thermal bremsstrahlung model to the pulsed X-ray flux.

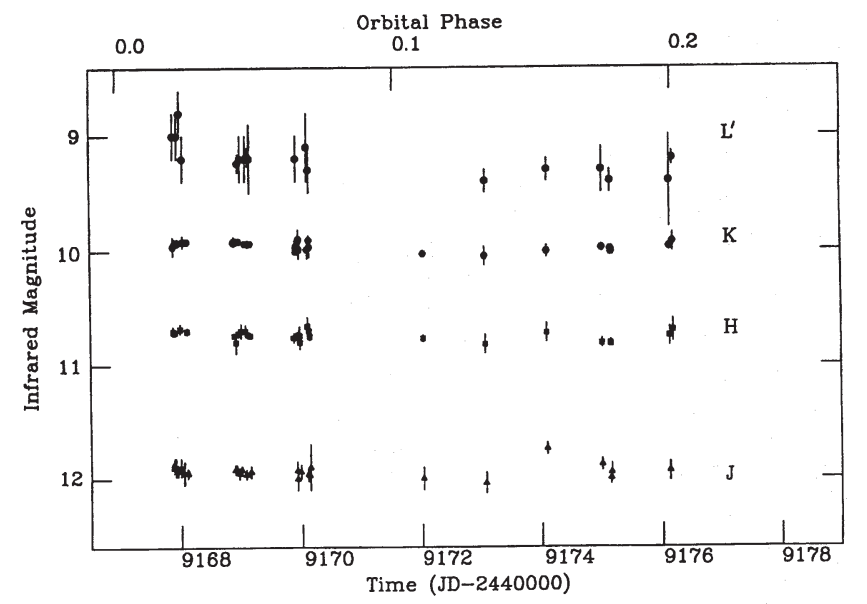

Figure 4. The $J$-, $H$-, $K$-, and $L^{\prime}$-band light curves of EXO2030+375 during the X-ray outburst.

it is not possible to say whether this represents a change that is correlated with orbital phase or merely a secular change in the Be star envelope. To examine the possibility of longterm infrared changes, we show in Table 3 data obtained over the last eight years (1985-1993), all from UKIRT service observations. Table 3 also includes the average magnitudes from Table 2, during the outburst campaign.
Table 2. UKIRT photometric observation log and infrared magnitudes.

$\begin{array}{lrrrrr}\text { JD } & \text { Orbital } & J & H & K & L^{\prime} \\ 2440000+ & \text { Phase } & & & & \\ & & & & & \\ 9161.064 & 0.872 & 12.04 & 10.82 & 10.02 & 9.21 \\ 9167.874 & 0.020 & 11.90 & 10.70 & & \\ 9167.880 & 0.020 & 11.87 & 10.71 & 9.96 & 9.0 \\ 9167.908 & 0.021 & 11.90 & 10.71 & 9.94 & \\ 9167.945 & 0.022 & 11.93 & & 9.93 & 9.0 \\ 9167.994 & 0.023 & 11.90 & 10.68 & 9.93 & 8.8 \\ 9168.040 & 0.024 & 11.95 & & 9.92 & \\ 9168.047 & 0.024 & 11.95 & & & 9.2 \\ 9168.106 & 0.025 & 11.95 & 10.70 & 9.92 & \\ 9168.892 & 0.042 & 11.92 & 10.74 & 9.93 & \\ 9168.915 & 0.042 & 11.92 & 10.80 & 9.92 & \\ 9168.957 & 0.043 & 11.95 & 10.72 & 9.92 & 9.2 \\ 9168.997 & 0.044 & 11.92 & 10.70 & & 9.2 \\ 9169.073 & 0.046 & 11.96 & 10.70 & 9.94 & 9.2 \\ 9169.117 & 0.047 & & 10.73 & 9.94 & 9.2 \\ 9169.148 & 0.047 & 11.94 & 10.74 & 9.94 & 9.2 \\ 9169.874 & 0.064 & & 10.76 & & \\ 9169.922 & 0.065 & 11.93 & 10.74 & 9.99 & 9.2 \\ 9169.927 & 0.065 & 12.00 & & 9.95 & \\ 9169.951 & 0.066 & & 10.73 & 9.92 & \\ 9169.980 & 0.066 & 11.94 & 10.77 & 9.95 & \\ 9170.101 & 0.069 & 11.96 & 10.66 & 9.99 & 9.1 \\ 9170.126 & 0.069 & 11.97 & 10.70 & 9.91 & \\ 9170.137 & 0.070 & 11.90 & 10.75 & 9.97 & 9.3 \\ 9172.024 & 0.110 & 12.00 & 10.77 & 10.03 & \\ 9173.068 & 0.133 & 12.04 & 10.82 & 10.05 & 9.4 \\ 9174.091 & 0.155 & 11.74 & 10.72 & 10.01 & 9.3 \\ 9175.003 & 0.175 & 11.88 & 10.81 & 9.98 & 9.3 \\ 9175.146 & 0.179 & 12.00 & 10.81 & 9.99 & \\ 9175.155 & 0.179 & 12.03 & 10.82 & 10.01 & 9.4 \\ 9176.121 & 0.199 & 11.95 & 10.75 & 9.97 & 9.4 \\ 9176.128 & 0.199 & 11.94 & & & \\ 9176.180 & 0.200 & & 10.70 & 9.93 & 9.2 \\ 9210.829 & 0.953 & 12.21 & 10.95 & 10.10 & 9.26\end{array}$

Table 3. Long-term infrared photometry of EXO2030+375.

$\begin{array}{lcrrrr}\text { JD } & \begin{array}{c}\text { Orbital } \\ 2440000+\end{array} & J & H & K & L^{\prime} \\ & \begin{array}{l}\text { Phase } \\ 6269\end{array} & & & & \\ 6714 & 0.96 & 11.87 & 10.63 & 9.75 & \\ 6974 & 0.29 & 11.89 & 10.66 & 9.81 & 9.04 \\ 7026 & 0.64 & 11.91 & 10.67 & 9.81 & 9.01 \\ 7105 & 0.51 & 11.97 & 10.68 & 9.85 & 9.11 \\ 8565 & 0.80 & 11.92 & 10.63 & 9.79 & 9.06 \\ 8741 & 0.08 & 11.93 & 10.64 & 9.83 & 8.99 \\ 8852 & 0.25 & 11.84 & 10.58 & 9.74 & 8.89 \\ 9161 & 0.16 & 11.94 & 10.64 & 9.79 & 8.98 \\ 9168-9176 & 0.87 & 12.04 & 10.82 & 10.02 & 9.21 \\ 9211 & 0.02-0.20 & 11.94 & 10.74 & 9.96 & 9.20 \\ & 0.95 & 12.21 & 10.95 & 10.10 & 9.26\end{array}$

As can be seen, the magnitudes in 1993 August are the faintest we have observed from EXO2030+375 over the last eight years. The system has been observed at many different orbital phases over this period, but no correlation of magnitude changes with orbital phase is apparent. We therefore conclude that any changes in the infrared magnitudes are not related to the motion of the neutron star and any X-ray outbursts that occur.

As a comparison with these infrared data, we note that

\section{Royal Astronomical Society • Provided by the NASA Astrophysics Data System}


service observations obtained at the 1-m Jacobus Kapteyn telescope (JKT), La Palma, on 1993 June 20 (JD 244 9159) revealed $B=22.4 \pm 0.4$ and $V=19.39 \pm 0.05$. These data were obtained with the EEV7 CCD, and transformed to the Johnson system using observations of the Landolt standard 111z717 (Landolt 1983). Previous observations for the Be star in the EXO2030+375 system gave $B=23.0 \pm 0.5$ and $V=19.68 \pm 0.08$ (Motch \& Janot-Pacheco 1987). Hence the recent data suggest a long-term brightening at optical wavelengths coinciding with the recent fade seen in the infrared.

\subsubsection{Polarimetry}

Full details of the reduction procedure applicable to IRCAM polarimetry are given by Aspin et al. (1990), but an outline of it is as follows. The polarimetric images were dark-fieldsubtracted and flat-fielded as above, and then realigned, as slight shifts in the position of the star in the four images would cause an anomalous polarization. An 8-arcsec aperture was used to measure the polarized flux for EXO2030+375, a comparison star in the same field, the sky background polarization in the vicinity of EXO2030+375, and images of S106, the polarimetric standard. Regions II and III of the S106 nebula were used for calibration, as these are reflection nebulae and the position angle of their polarization is assumed to be stable (Aspin, private communication). This processing was performed on all four polarization images to produce four intensity measurements for the target, comparison and standard objects on each night. The intensities from the four images are here called $I_{0}, I_{45}, I_{22.5}$ and $I_{67.5}$ and the normalized Stokes parameters of linear polarization $(Q$ and $U$ ) were calculated using the formulae

$$
\begin{aligned}
& Q(\%)=100 \times\left[\left(I_{0}-I_{45}\right) /\left(I_{0}+I_{45}\right)\right], \\
& U(\%)=100 \times\left[\left(I_{22.5}-I_{67.5}\right) /\left(I_{22.5}+I_{67.5}\right)\right] .
\end{aligned}
$$

The percentage polarization, $P$, and the position angle of polarization, $\theta$, were then calculated using

$P=\left(Q^{2}+U^{2}\right)^{1 / 2}$,

$\theta=\frac{1}{2} \arctan (U / Q)$.

The results obtained from the five nights of polarimetric observations are shown in Table 4 . The (raw) position angles are accurate to $\sim 2^{\circ}$ and the individual values for polarized fraction are accurate to $\sim 1$ per cent. The average polarization of the sky was found to be 1.0 per cent in the $J$ band and 0.2 per cent in the $K$ band. This contribution has not been removed from the data listed in Table 4.

It was found that there was a systematic shift of about $30^{\circ}$ in the measured position angle between the observations made on the first three nights and those made on the final two nights. This is because IRCAM was removed between the two sessions and the waveplate was not realigned on replacement. This shift has been taken account of using the standard (S106) observations, but, because of anomalies between the correction angles derived from the different regions of the nebula, there is a systematic error of $\pm 5^{\circ}$ between the position angle results at the beginning and the end of the campaign. The position angle corrections calculated from the observations of S106 were $+45^{\circ} \pm 5^{\circ}$ for measurements on the first three nights and
Table 4. Polarimetry results.

$\begin{array}{lllll}\text { JD } & \text { Filter } & \begin{array}{l}\text { polarized } \\ \text { fraction }\end{array} & \begin{array}{l}\text { Raw } \\ \text { pos. } \\ \text { angle }\end{array} & \begin{array}{l}\text { Corr. } \\ \text { pos. } \\ \text { angle }\end{array} \\ \text { EXO2030+375: } & & & & \\ 9167.986 & J & 9.7 \% & 9^{\circ} & 54^{\circ} \\ 9168.118 & J & 10.0 \% & 8^{\circ} & 53^{\circ} \\ 9168.938 & J & 9.9 \% & 13^{\circ} & 58^{\circ} \\ 9170.083 & J & 10.3 \% & 14^{\circ} & 59^{\circ} \\ 9175.021 & J & 9.2 \% & 42^{\circ} & 59^{\circ} \\ 9176.146 & J & 11.1 \% & 43^{\circ} & 60^{\circ} \\ & & & & \\ 9168.118 & K & 4.0 \% & 6^{\circ} & 51^{\circ} \\ 9168.938 & K & 4.3 \% & 12^{\circ} & 57^{\circ} \\ 9170.083 & K & 4.0 \% & 12^{\circ} & 58^{\circ} \\ 9175.021 & K & 4.6 \% & 38^{\circ} & 55^{\circ} \\ 9176.146 & K & 4.3 \% & 44^{\circ} & 61^{\circ} \\ & & & & \\ \text { Average } & J & 10.0 \pm 0.6 \% & & 57^{\circ} \pm 3^{\circ} \\ & K & 4.2 \pm 0.2 \% & & 56^{\circ} \pm 3^{\circ} \\ \text { comparison star : } & & & & \\ 9170.083 & J & 5.2 \% & 28^{\circ} & 73^{\circ} \\ 9175.021 & J & 5.1 \% & 44^{\circ} & 61^{\circ} \\ 9176.146 & J & 6.0 \% & 40^{\circ} & 57^{\circ} \\ & & & & \\ 9170.083 & K & 2.5 \% & 8^{\circ} & 53^{\circ} \\ 9175.021 & K & 2.3 \% & 34^{\circ} & 51^{\circ} \\ 9176.146 & K & 2.5 \% & 40^{\circ} & 57^{\circ} \\ & & & & 64^{\circ} \pm 7^{\circ} \\ \text { Average } & J & 5.4 \pm 0.4 \% & & 53^{\circ} \pm 2^{\circ} \\ & K & 2.4 \pm 0.1 \% & & \end{array}$

$+17^{\circ} \pm 5^{\circ}$ for measurements on the last two nights. These values have been used to convert raw position angles to corrected position angles in Table 4.

The polarized fraction and angles measured were consistent over the five nights' observations for both EXO2030+375 and the comparison star in the same field. Hence we show in Table 4 the average of the preceding values in the table, along with the $1 \sigma$ scatter, for these two objects. Several other sources were observed that showed negligible polarization.

\subsection{Optical data}

The spectroscopic data from both Palomar and the WHT were reduced using the FIGARO software package (Shortridge 1991; Meyerdierks 1992). After bias frame subtraction and flat-fielding, the spectra were extracted and a sky (background) spectrum from an adjacent region of the field was subtracted. Wavelength calibration was performed using an appropriate arc, and the spectra rebinned to a linear wavelength range. The spectra of EXO2030+375 were all flux-calibrated with respect to the standard star, and corrected for airmass changes using average extinction curves for the respective sites (except for the spectrum obtained on 1993 June 24, for which no flux calibration was possible).

The main aim of the spectroscopy was to monitor any changes in the $\mathrm{H} \alpha$ and $\mathrm{H} \beta$ lines from the Be star. Unfortunately, the counterpart to EXO2030+375 is so reddened that it is extremely faint in the blue spectral region $(B \sim 23)$, and as a result no significant detections were obtained of the $\mathrm{H} \beta$ line. However, the $\mathrm{H} \alpha$ line was detected in emission, as expected, 
Table 5. Line parameters fitted to nightly mean and overall mean $\mathrm{H} \alpha$ spectra.

$\begin{array}{llllll}\begin{array}{l}\text { JD } \\ 2440000+\end{array} & \begin{array}{l}\text { Line } \\ \text { centre } \\ (\AA)\end{array} & \begin{array}{l}\text { Peak } \\ \text { height } \\ (\mathrm{mJy})\end{array} & \text { FWHM } & \begin{array}{l}\text { Line } \\ \text { flux } \\ (\mathrm{mJy} \AA)\end{array} & \begin{array}{l}\text { Equiv. } \\ \text { width } \\ (\AA)\end{array} \\ 9163.7, & 6561.5 & - & 10.8 & - & -16.7 \\ 9168.6 & 6562.4 & 0.21 & 11.8 & 2.6 & -15.4 \\ 9169.0 & 6561.7 & 0.29 & 7.4 & 2.3 & -18.3 \\ 9169.5 & 6562.0 & 0.23 & 11.7 & 2.9 & -15.1 \\ 9168-9170 & 6562.2 & 0.22 & 10.7 & 2.5 & -14.5\end{array}$

and its behaviour examined over three nights on the rise of the X-ray outburst.

The $\mathrm{H} \alpha$ emission lines in each of the medium-resolution spectra were fitted using the GAUSS routine in FIGARO. There were hints that a single Gaussian line did not provide a good fit to the $\mathrm{H} \alpha$ line in several of the spectra and, in some of these cases, fits using two Gaussian lines provided marginally more acceptable results. However, no spectra show clear evidence for a splitting of the peak into blue and red wings, so it is not clear that a fit using two Gaussian lines is justified. The results from fitting both single and double Gaussian lines to all the spectra showed apparent variations in all of the line parameters over the three nights of data. Variations in peak height, line width and equivalent width amount to about \pm 25 per cent of the mean values. However, these variations are random and are not correlated with the gradual increase in X-ray flux that is seen during this time (Fig. 1). We conclude that the apparent variations seen are probably due to the poor signal-to-noise ratio of the spectra. If they are real, the variations are most likely due to changes in the structure of the circumstellar envelope that are unrelated to the passage of the neutron star. For this reason, we show in Fig. 5 only the mean $\mathrm{H} \alpha$ spectrum. This line profile is symmetric and can be well fitted using a single Gaussian. The parameters obtained from the fit to this mean spectrum are shown in Table 5. This table also shows parameters fitted to the mean of each night's spectra and the pre-outburst service spectrum. The fact that the $\mathrm{H} \alpha$ equivalent width measured from the overall mean spectrum is lower than those measured in all three nightly mean spectra should not cause concern. It probably indicates that certain features in the $\mathrm{H} \alpha$ wings of the nightly mean spectra become averaged out in the overall mean spectrum. It may therefore be a marginal indication of a change in the $\mathrm{H} \alpha$ emission properties, but we would not like to read too much into this.

\section{DISCUSSION}

We observed no change in either the infrared photometric magnitudes or the $\mathrm{H} \alpha$ line profile and strength during this cataclysmic event, despite an increase in the pulsed X-ray flux by roughly an order of magnitude. That is to say, the passage of the neutron star through the circumstellar material around the Be star has no detectable effect on the structure or properties of that material.

There are two ways in which the passage of the neutron star through the circumstellar material of the Be star may be expected to influence the emission properties of that material. First, the intense flux of X-rays produced as a result of ac- cretion on to the neutron star will be partially intercepted by the circumstellar material. This X-ray flux may therefore be reprocessed into optical or infrared emission. Since the signature of the circumstellar material is its $\mathrm{H} \alpha$ line and infrared continuum emission, any reprocessed X-ray flux may be expected to alter this signature. Secondly, the passage of the neutron star itself may disrupt the physical structure of the circumstellar envelope as it accretes from it. This may give rise to a change in the emission characteristics of this material that would, again, be most obviously seen as a change in the Balmer emission lines and the infrared luminosity. The fact that no such changes are observed implies that neither of these effects is in fact significant, at the levels to which we are sensitive. These scenarios are discussed in more detail below, where some approximate values are placed on the the sizes of these effects.

Another possibility is that an accretion disc and HII region may form around the neutron star, and this too may become a source of $\mathrm{H} \alpha$ line emission during the X-ray outburst. Such a possibility has been discussed by Apparao (1991) and we consider this below also.

\subsection{Reprocessed X-ray emission}

We can estimate a limit for the fraction of the X-ray emission that is reprocessed into $\mathrm{H} \alpha$ or infrared emission, given that we see no correlation between the rising X-ray flux during the outburst and the $\mathrm{H} \alpha$ line or infrared flux.

\subsubsection{The infrared continuum}

We take the $K$ band as an example; the results in the other infrared bands are similar but less pronounced. Using the interstellar extinction law of Rieke \& Lebofsky (1985) and the value $E(B-V)=3.8$ for $\mathrm{EXO} 2030+375$ (Motch \& JanotPacheco 1987), we obtain $A_{K}=1.3 \mathrm{mag}$. Hence the $K$-band flux produced in the circumstellar environment is reduced by a factor of 3.3 at the Earth. Now, the mean $K$-band magnitude observed during the X-ray outburst, $K=10.0$, corresponds to a flux of $2.3 \times 10^{-11} \mathrm{erg} \mathrm{s}^{-1} \mathrm{~cm}^{-2}$ at $2.2 \mu \mathrm{m}$ (calculated using Johnson's standard absolute calibration of filter bands as given by Zombeck (1990) and assuming a bandpass of $1.9 \mu \mathrm{m}$ to $2.5 \mu \mathrm{m})$. As a conservative estimate, we assume that a systematic change of $0.1 \mathrm{mag}$ in $K$ would be detectable in these observations. This equates to a change in $K$-band flux of about 10 per cent. Since we see no such systematic change, we can determine an upper limit to the $\mathrm{X}$-ray flux that is reprocessed into infrared emission, $F_{K}$, namely, $F_{K} \leqslant\left(3.3 \times 2.3 \times 10^{-11} \times 0.1\right) \mathrm{erg} \mathrm{s}^{-1} \mathrm{~cm}^{-2}$. So $F_{K} \leqslant 7.6 \times 10^{-12} \mathrm{erg} \mathrm{s}^{-1} \mathrm{~cm}^{-2}$. We therefore conclude that at most 0.7 per cent of the observed pulsed X-ray flux is reprocessed into infrared emission by the circumstellar material.

\subsubsection{The $H \alpha$ line}

An upper limit to the fraction of X-ray emission that is reprocessed into the $\mathrm{H} \alpha$ line can be calculated following a similar argument to that used above. The wavelength of $\mathrm{H} \alpha$ falls between the Johnson $V$ and $R$ bands, so we can estimate the reddening at $6563 \AA$ as $A_{\mathrm{H} \alpha}=\left(A_{V}+A_{R}\right) / 2$. Again, using the interstellar extinction law of Rieke $\&$ Lebofsky (1985) and the 
EX02030+375 MEAN SPECTRUM

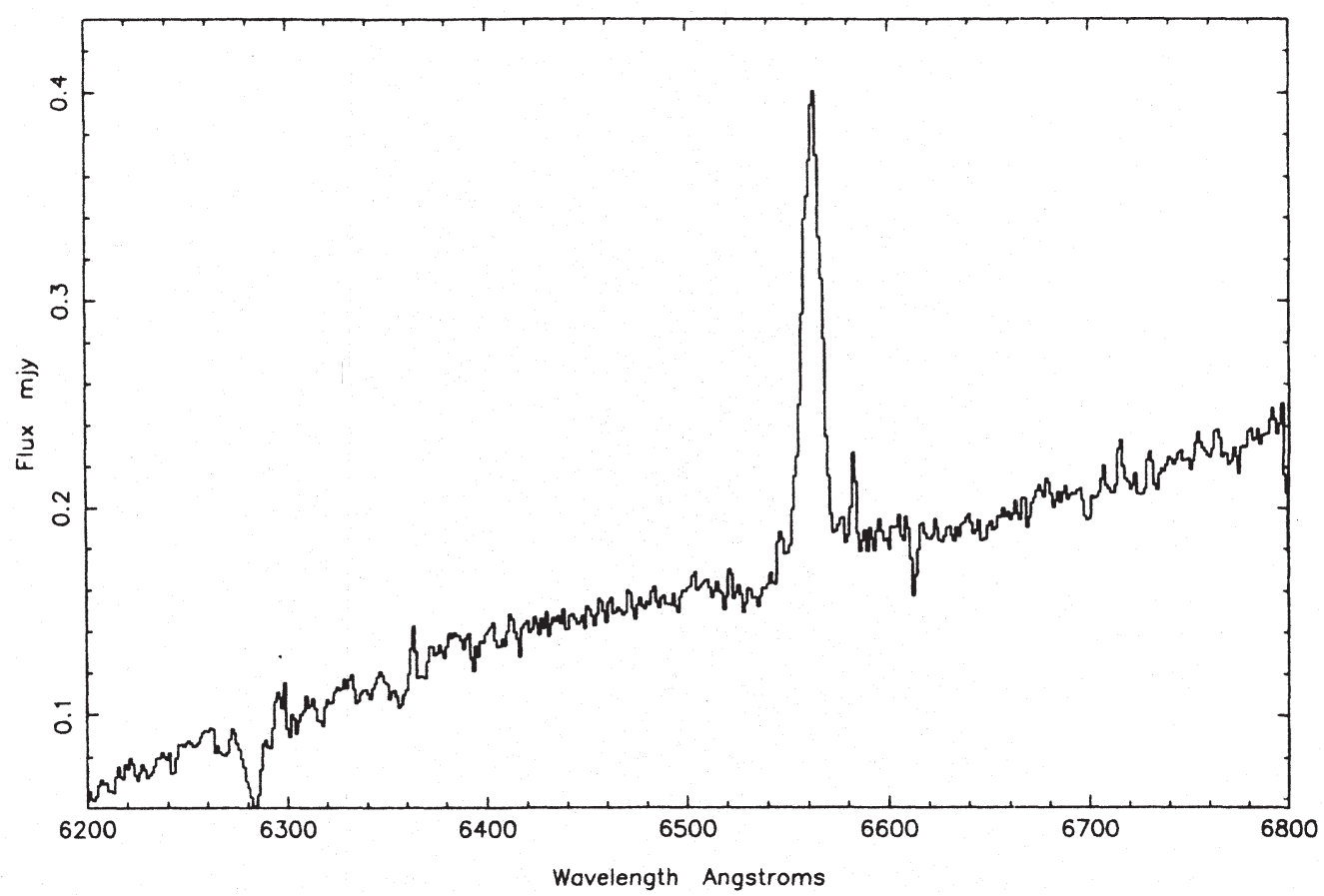

Figure 5. The average medium-resolution $\mathrm{H} \alpha$ spectrum of EXO2030+375 obtained at the WHT and Palomar on 1993 June $29 / 30$.

value $E(B-V)=3.8$ for EXO2030+375 (Motch \& JanotPacheco 1987), we obtain $A_{\mathrm{H} \alpha}=10.3 \mathrm{mag}$. Hence the $\mathrm{H} \alpha$ flux produced in the circumstellar environment is reduced by a factor of $\sim 13 \times 10^{3}$ at the Earth. Now, the mean $\mathrm{H} \alpha$ line flux seen in the observations here is about $2.5 \mathrm{mJy} \AA$ (Table 5) which corresponds to $1.75 \times 10^{-15} \mathrm{erg} \mathrm{s}^{-1} \mathrm{~cm}^{-2}$ at $6563 \AA$. We assume that a systematic change in the $\mathrm{H} \alpha$ line flux by about 25 per cent would be detectable over the course of our observations, given the random fluctuations that are observed. Since we see no such systematic change, an upper limit to the $\mathrm{X}$-ray flux that is reprocessed into the $\mathrm{H} \alpha$ line, $F_{\mathrm{H} \alpha}$, is given by $F_{\mathrm{H} \alpha} \leqslant\left(13 \times 10^{3} \times 1.75 \times 10^{-15} \times 0.25\right) \mathrm{erg} \mathrm{s}^{-1} \mathrm{~cm}^{-2}$. So $F_{\mathrm{H} \alpha} \leqslant 5.7 \times 10^{-12} \mathrm{erg} \mathrm{s}^{-1} \mathrm{~cm}^{-2}$. We therefore conclude that at most 0.5 per cent of the observed pulsed X-ray flux is reprocessed into $\mathrm{H} \alpha$ emission by the circumstellar material.

\subsection{Disruption of the circumstellar material}

\subsubsection{Orbital parameters}

The orbital parameters derived from the long-term BATSE light curve of EXO2030+375 allow us to derive the mass function of the neutron star, namely

$\frac{4 \pi^{2}\left(a_{\mathrm{x}} \sin i\right)^{3}}{G P_{\text {orb }}^{2}}=9.75_{-2.5}^{+3.0} \mathrm{M}_{\odot}=\frac{\left(M_{1} \sin i\right)^{3}}{\left(M_{1}+M_{\mathrm{x}}\right)^{2}}$,

where $M_{1}$ is the mass of the Be star and $M_{x}$ is the mass of the neutron star. If we assume that $M_{\mathrm{x}}=1.44 \mathrm{M}_{\odot}$, then we can solve the mass function for $M_{1}$ as a function of $i$, the inclination angle. Taking the spectral type of the Be star to be around BOV (Coe et al. 1988), it should have a mass of the order of $18 \pm 2 \mathrm{M}_{\odot}$. This in turn implies that the system inclination angle, $i=60^{\circ}+20^{\circ}$. Hence the semi-major axis of the neutron star's orbit, $a_{\mathrm{x}}=310_{-70}^{+80}$ light seconds. With these estimates for $i, M_{1}$ and $M_{\mathrm{x}}$, we can also estimate the size of the semi-major axis of the Be star's orbit, namely $a_{1} \sim 25$ light seconds. Hence the separations between the neutron star and the Be star at apastron and periastron, given by $\left(a_{\mathrm{x}}+a_{1}\right)(1 \pm e)$, are then $26 R_{1}$ and $13 R_{1}$ respectively, where the radius of the Be star, $R_{1}=7.4 R_{\odot}=5.15 \times 10^{9} \mathrm{~m}$. (The mass and radius of a B0V star are taken from Zombeck (1990).)

\subsubsection{Accretion rates}

Using the estimated outburst flux, $F_{\mathrm{x}}=1.15 \times$ $10^{-9} \mathrm{erg} \mathrm{cm}^{-2} \mathrm{~s}^{-1}$, and the distance to the source derived by Parmar et al. (1989a), $D=5 \mathrm{kpc}$, we obtain an outburst luminosity, $L_{\mathrm{x}}=3.4 \times 10^{36} \mathrm{erg} \mathrm{s}^{-1}$. Now, assuming all the gravitational energy is converted into X-rays during the accretion process, we can put $L_{\mathrm{x}}=L_{\mathrm{acc}}=G M_{\mathrm{x}} \dot{M} / R_{\mathrm{x}}$. Hence we obtain a mass accretion rate, $\dot{M}=1.8 \times 10^{16} \mathrm{~g} \mathrm{~s}^{-1}$. This assumes a neutron star mass, $M_{\mathrm{x}}=1.44 \mathrm{M}_{\odot}$, and radius, $R_{\mathrm{x}}=10 \mathrm{~km}$. Since EXO2030+375 produces this X-ray intensity for about 1 week, this corresponds to a total accreted mass, $M_{\text {acc }} \sim 10^{22} \mathrm{~g}$.

We now proceed to estimate the total mass of the $\mathrm{H} \alpha$ emission region in the circumstellar envelope. These figures are necessarily only an order of magnitude estimate but are useful in determining whether the neutron star outburst may be expected to influence the emission properties of the circumstellar material. The $\mathrm{H} \alpha$ emission line is generally found to arise in a region situated about 10 stellar radii from the Be star (Slettebak, Collins \& Truax 1992), and the opening angle for the circumstellar disc of material is estimated to be about 0.5 in Be stars (Bjorkmann \& Cassinelli 1993). The circumstellar disc is therefore about $0.1 R_{1}$ thick at the radius where the $\mathrm{H} \alpha$ line forms. If we assume that the bulk of the $\mathrm{H} \alpha$ emission 
arises in an annular slab of width $\approx 10 R_{1}$ centred on $10 R_{1}$, then the neutron star would pass within this region at periastron (see estimates for stellar separations above). This gives a total volume for the $\mathrm{H} \alpha$ emission region as $\approx 10^{37} \mathrm{~cm}^{3}$. A typical density for the circumstellar material in the vicinity of the $\mathrm{H} \alpha$ emission region is of the order of $\approx 10^{12}$ atom $\mathrm{cm}^{-3}$ (Slettebak 1979). Hence the mass of the $\mathrm{H} \alpha$ emission region is $\approx 10^{25} \mathrm{~g}$.

So the mass accreted by the neutron star during the course of the outburst is of the order of 0.1 per cent of the total mass of the $\mathrm{H} \alpha$ emission region. Hence it is perhaps no surprise that we see no change in the properties of the $\mathrm{H} \alpha$ emission line during this event, since the major part of the region producing the line remains intact during the passage of the neutron star.

\subsection{Emission from the vicinity of the neutron star}

The X-rays emitted from the neutron star will form an HII region in the envelope of the Be star which then emits line and continuum radiation. Apparao (1991) has calculated the strength of $\mathrm{H} \alpha$ line emission that would be produced by such a process for a range of X-ray spectra and luminosities. Note however that Apparao assumes the additional $\mathrm{H} \alpha$ emission to form in a spherically symmetric region around the neutron star. Currently, 'thin' circumstellar discs are the most popular explanation for the $\mathrm{Be}$ star phenomenon (e.g. $0.1 R_{1}$ thick at $10 R_{1}$ from the Be star (Bjorkmann \& Cassinelli 1993)). Hence it is not certain that the HII region produced by the neutron star, as envisioned by Apparao, will lie wholly within the circumstellar disc of the Be star. In this case, the comparison below may not be entirely valid.

The neutron star in EXO2030+375 produces an X-ray luminosity of $3.4 \times 10^{36} \mathrm{erg} \mathrm{s}^{-1}$, assuming a distance of $5 \mathrm{kpc}$, and has a bremsstrahlung spectrum with a temperature of about $20 \mathrm{keV}$ during the outburst. Apparao lists the results for parameter values $k T=10 \mathrm{keV}$ with $L_{\mathrm{x}}=10^{38}, 10^{37}, 10^{36}$ and $10^{35} \mathrm{erg} \mathrm{s}^{-1}$, but unfortunately not for any harder spectra. The $\mathrm{H} \alpha$ emission line luminosity produced by the HII region around the neutron star scales such that $L_{\mathrm{x}} \propto\left(L_{\mathrm{H} \alpha}\right)^{2 / 3}$. Hence, interpolating between the results given by Apparao, we calculate that the HII region produced around the neutron star in EXO2030+375 during this outburst will produce an additional $\mathrm{H} \alpha$ line luminosity of $\sim 3.5 \times 10^{34} \mathrm{erg} \mathrm{s}^{-1}$. (N.B. EXO2030+375 has a harder spectrum than the $10 \mathrm{keV}$ modelled by Apparao, so this may cause a slight decrease in the $\mathrm{H} \alpha$ luminosity calculated above.)

Now, the $\mathrm{H} \alpha$ line flux observed during the outburst is $\sim 1.75 \times 10^{-15} \mathrm{erg} \mathrm{s}^{-1} \mathrm{~cm}^{-2}$ (Section 4.1.2). As noted earlier, interstellar reddening causes the $\mathrm{H} \alpha$ flux emitted by EXO2030+375 to be reduced by a factor $13 \times 10^{3}$ by the time it reaches us. Hence the $\mathrm{H} \alpha$ line luminosity of $\mathrm{EXO} 2030+375$ is calculated as $\sim 7 \times 10^{34} \mathrm{erg} \mathrm{s}^{-1}$ during the outburst, assuming a distance of $5 \mathrm{kpc}$. So the additional $\mathrm{H} \alpha$ line flux produced in the vicinity of the neutron star should be about 50 per cent of that observed. As EXO2030+375 approaches or recedes from outburst we may therefore expect to see $\mathrm{H} \alpha$ line intensity changes by up to 50 per cent. Since we do not see such an effect, this suggests that the values calculated by Apparao may not be appropriate to EXO2030+375, possibly for the reason mentioned above.

\subsection{Wind velocities}

The X-ray flux we have obtained and the accurate orbital parameters derived from the BATSE long-term light curve allow us to make estimates of the velocity of the wind outflowing from the Be star. The discussion here follows the derivation presented by Waters et al. (1988).

The mass accretion rate on to the neutron star is given by:

$\dot{M}=\frac{4 \pi G^{2} M_{\mathrm{x}}^{2} F_{\mathrm{m}}}{v_{\mathrm{rel}}^{4}}$

where $v_{\text {rel }}$ is the relative velocity between the neutron star and the material that it accretes and $F_{\mathrm{m}}$ is the mass flux at the distance from the Be star at which accretion occurs. Assuming that all the gravitational energy is converted into X-rays, we have:

$L_{\mathrm{x}}=\frac{4 \pi G^{3} M_{\mathrm{x}}^{3} F_{\mathrm{m}}}{R_{\mathrm{x}} v_{\mathrm{rel}}^{4}}$.

The relative velocity between the neutron star and the circumstellar wind is given by:

$v_{\text {rel }}^{2}=v_{\text {wind }}^{2}+v_{\text {orb }}^{2}$

where $v_{\text {wind }}$ and $v_{\text {orb }}$ are the radial outflow velocity of the circumstellar wind and the orbital velocity of the neutron star respectively, at the position in the orbit in question. The orbital velocity of the neutron star at a given distance, $r$, from the $\mathrm{Be}$ star is given by:

$v_{\text {orb }}^{2}=G\left(M_{1}+M_{\mathrm{x}}\right)\left(\frac{2}{r}-\frac{1}{a}\right)$

where $a=a_{\mathrm{x}}+a_{1}$. From this equation we obtain neutron star velocities at periastron and apastron of $\sim 230 \mathrm{~km} \mathrm{~s}^{-1}$ and $\sim 110 \mathrm{~km} \mathrm{~s}^{-1}$ respectively, under the assumptions above. To continue, the mass outflow rate can be written as:

$F_{\mathrm{m}}=\rho_{0} v_{0}\left(\frac{R_{1}}{r}\right)^{2}\left(1+\frac{v_{\text {orb }}^{2}}{v_{\text {wind }}^{2}}\right)^{1 / 2}$

where $\rho_{0}$ and $v_{0}$ are the density and outflow velocity of the wind at the surface of the Be star, and $r$ is an arbitrary radial distance from the star's surface. Hence, substituting for $v_{\text {rel }}$ and $F_{\mathrm{m}}$ into equation (7), and setting $r$ equal to the periastron distance of the neutron star, $a(1-e)$, we obtain:

$L_{\mathrm{x}}=\frac{4 \pi G^{3} M_{\mathrm{x}}^{3} R_{1}^{2} \rho_{0} v_{0}}{R_{\mathrm{x}} a^{2}(1-e)^{2} v_{\text {wind }}^{4}}\left(1+\frac{v_{\text {orb }}^{2}}{v_{\text {wind }}^{2}}\right)^{-3 / 2}$.

Now, using the parameters from earlier, namely $L_{\mathrm{x}}=3.4 \times$ $10^{36} \mathrm{erg} \mathrm{s}^{-1}, M_{\mathrm{x}}=1.44 \mathrm{M}_{\odot}, R_{\mathrm{x}}=10 \mathrm{~km}, a=340$ light seconds, $e=0.33, R_{1}=7.4 \mathrm{R}_{\odot}, v_{\text {orb }}=230 \mathrm{~km} \mathrm{~s}^{-1}, v_{0}=5 \mathrm{~km} \mathrm{~s}^{-1}$ and $\rho_{0}=10^{-11} \mathrm{~g} \mathrm{~cm}^{-3}$ (these last two being typical values as used by Waters et al. (1988)), we can solve the equation above to obtain the wind velocity at the radius corresponding to the distance of the neutron star at periastron. We obtain $v_{\text {wind }}=230 \mathrm{~km} \mathrm{~s}^{-1}$ at $r=13 R_{1}$. If we now consider the case at apastron, the $\mathrm{X}$-ray luminosity is reduced to $<10$ per cent of that at periastron (Fig. 1) and $v_{\text {orb }}=110 \mathrm{~km} \mathrm{~s}^{-1}$. We replace $a(1-e)$ by $a(1+e)$, the apastron distance, and solve the equation to obtain $v_{\text {wind }}>360 \mathrm{~km} \mathrm{~s}^{-1}$ at $r=26 R_{1}$. These figures are as expected, both in magnitude and in their relative values, for the outflow velocity of an equatorial wind from a Be star (Waters et al. 1988). We note also that these velocities 
support the Waters et al. relationship, derived from infrared observations of several Be star/X-ray binaries, that $v_{\text {wind }} \propto r$, if the X-ray flux at apastron is about 4 per cent of the X-ray flux at periastron. This is fully consistent with the X-ray light curve seen with BATSE (Fig. 1).

\subsection{Implications of the polarimetry results}

Poeckert, Bastien \& Landstreet (1979) detected polarization in about half of the Be stars they observed (all below 2 per cent at $V$ ). This polarization is caused by Thomson scattering from free electrons and decreases sharply across the Balmer and then the Paschen series. Since the polarization will be lower at longer wavelengths, it is unlikely that the polarization we have seen in the infrared emission from EXO2030+375 can be explained in this way. Intrinsic polarization may also be caused by Mie scattering from dust grains aligned in a magnetic field about the star. As there is no evidence for large amounts of dust in the system, it is unlikely that this process can explain the observed polarization either. Bearing this in mind, we examine below whether or not the observed polarization could arise in the interstellar medium.

\subsubsection{Interstellar polarization}

Serkowski, Mathewson \& Ford (1975) put an upper limit on the interstellar polarized fraction as a function of the extinction, namely $P_{\max }=9.0 E(B-V)$. For $E(B-V)=3.8$ as in EXO2030+375, this implies $P_{\max }=34$ per cent. We note, however, that the $U B V R I$ photometry data used to determine this law only included objects up to $E(B-V) \sim 1$, and appear to turn over at 7 per cent. None the less, interstellar polarizations over 10 per cent at $J$ have been observed in $\mathrm{OH} / \mathrm{IR}$ stars by Jones \& Gehrz (1990). Hence the polarization we have measured could feasibly be interstellar in origin.

We can investigate the above hypothesis using a method similar to that used by Jones \& Gehrz (1990). Adapting a formula from their work we obtain:

$$
\frac{P_{J}}{P_{K}}=\frac{\exp \left[-1.7 \lambda_{\max } \ln ^{2}\left(\lambda_{\max } / \lambda_{J}\right)\right]}{\exp \left[-1.7 \lambda_{\max } \ln ^{2}\left(\lambda_{\max } / \lambda_{K}\right)\right]}
$$

where $\lambda_{\max }$ is the wavelength at which the polarization is a maximum. Assuming that the polarization values for EXO2030+375 listed in Table 4 are entirely interstellar in origin, we can calculate $\lambda_{\max }$. As the values for $P_{J}$ and $P_{K}$ may be subject to a systematic error of $\sim 1$ per cent, the ratio $P_{J} / P_{K}=2.4_{-0.8}^{+1.5}$. This leads to a rather unconstrained value of $\lambda_{\max }=0.2_{-0.1}^{+1.2} \mu \mathrm{m}$. The value of $\lambda_{\max }$ has been shown by Whittet \& van Breda (1978) to be related to the ratio of total to selective extinction, $R=A_{V} / E(B-V)$, by $R=5.6 \lambda_{\max }$. This would imply $R=1.1_{-0.5}^{+6.7}$ for EXO2030+375, which encompasses the standard value, $R=3.09$ as obtained by Rieke \& Lebofsky (1985). On this evidence, therefore, it is possible that the measured polarization in EXO2030+375 is due to the interstellar medium.

We note, however, that because of the large uncertainty on these values there is still room for an intrinsic polarization of up to 3 per cent in the $J$ band and up to 1 per cent in the $K$ band. For this reason we briefly consider another possible cause of intrinsic polarization in Be star/X-ray binaries.

\subsubsection{Intrinsic polarization}

As another suggestion for intrinsic polarization, Gnedin et al. (1981) discuss infrared emission being attributed to protoncyclotron masering in the polar regions of accreting neutron stars. Masering occurs where there is a population inversion caused by Coulomb scattering off the electrons creating an anisotropy in the pitch angle. As a result, polarized radiation is emitted at the proton gyrofrequency. Their model predicts $L_{\mathrm{IR}} / L_{\mathrm{x}} \sim 0.01$ for an accreting neutron star with a magnetic field $B=10^{11} \mathrm{G}$. Higher magnetic fields may cause emission in the UV/optical (Kirk \& Stoneham 1982).

Approximately 3 per cent of the infrared flux we observe may be polarized due to an intrinsic component of polarized emission in EXO2030+375. If this intrinsic component is highly polarized (say at the 60 per cent level), then we can estimate that $\sim 5$ per cent of the total infrared emission is produced by a process such as the proton-cyclotron masering discussed by Gnedin et al. (1981). For a $J$ magnitude of 12 , a change in the infrared flux of 5 per cent would produce a 0.06 mag change, which is near or below our limits of detectability. Our results from infrared photometry and polarimetry are therefore consistent with the model presented by Gnedin et al. (1981).

\subsection{Comparison with other Be star/X-ray binaries}

As mentioned earlier, the Be star/X-ray binary A1118-616 was recently observed in a multiwavelength campaign soon after the onset of an X-ray outburst (Coe et al. 1994). The outburst was detected by BATSE in 1992 January, and optical, infrared and ultraviolet observations were performed soon after. In contrast to EXO2030+375, the optical and infrared fluxes were higher during the X-ray outburst than they were prior to this episode. The model for this system is that the neutron star is in a nearly circular orbit around the Be star and is usually just on the edge of the circumstellar material. As a result, the source displays a quiescent X-ray emission that is roughly constant. Occasionally the circumstellar disc becomes inflated, giving rise to increased optical and infrared emission. As a result, the neutron star passes through denser regions of the disc and an X-ray outburst is seen that peaks near periastron.

In $\mathrm{EXO} 2030+375$, the orbit is rather eccentric $(e=0.33)$ and as a result the 'normal' state of the system is for a regular $\mathrm{X}$-ray outburst on every periastron passage. These normal outbursts are therefore probably the result of the neutron star moving in towards the disc, whereas in A1118-616 they are the result of the disc moving out towards the neutron star. We note, however, that EXO2030+375 occasionally undergoes massive outbursts (as seen in 1985 by EXOSAT). These X-ray outbursts are presumably caused by inflation of the Be star circumstellar disc, and show no evidence for modulation at the orbital period (Parmar et al. 1989a). Hence both processes may occur in some systems, including EXO2030+375.

A few Be star/X-ray binaries show behaviour similar to A1118-616, namely a roughly constant X-ray flux that is enhanced occasionally, presumably due to inflation of the disc. The well-studied system X Persei falls into this category, for instance. Rather more systems have similar behaviour to EXO2030+375, that is, they show regular X-ray outbursts on each periastron passage. Systems such as A0535+26 and V0332 +53 fall into this category, and both have similar orbital 
eccentricities to EXO2030+375. It is of course conceivable that other ('isolated') Be stars may be harbouring (unseen) neutron stars in wide, circular orbits which never result in the neutron star encountering a dense enough region of the circumstellar disc to initiate detectable X-ray emission. None the less, such systems may be recognized if the Be star ever undergoes an episode of disc expansion sufficient to 'turn on' the neutron star as an X-ray source.

\section{CONCLUSION}

A typical X-ray outburst has been observed from the neutron star in EXO2030+375 and was seen to have no detectable effect on the emission properties of the circumstellar material surrounding the Be star. This implies that the fraction of the $\mathrm{X}$-ray emission that is reprocessed into infrared continuum or $\mathrm{H} \alpha$ line emission must be less than 1 per cent of the accretion luminosity. We have also demonstrated that any disruption of the circumstellar material by the passage of the neutron star is minimal. From the calculations of Apparao (1991) we may have expected there to be a detectable $\mathrm{H} \alpha$ line flux produced in an HII region around the accreting neutron star. The fact that we see no such change in the $\mathrm{H} \alpha$ line before and during the X-ray outburst may suggest that the situation described by Apparao is not directly applicable to this object.

In summary, the neutron star and the X-ray emission it produces do not influence the behaviour of the $\mathrm{Be}$ star to any great extent in EXO2030+375. However, this null result in combination with the X-ray data is informative, and we are still able to deduce certain parameters of the circumstellar material, such as the radial outflow velocity of the wind. For reasons such as this, Be star/X-ray binaries still provide one of the most useful methods of investigating the behaviour of Be stars, in ways that are simply not possible for isolated systems. The fact that the neutron star is such a non-intrusive probe makes it easier to transfer conclusions drawn about $\mathrm{Be}$ star/X-ray binaries to other, non-binary Be stars.

It is at present unclear whether the measured polarization in EXO2030+375 is entirely of interstellar origin, or whether there is an intrinsic component as well. If variability were detected in further observations, this would support the intrinsic explanation and, if there were a drop in the polarization outside X-ray outburst, this would support the proton-cyclotron emission process.

It is anticipated that further studies of other (brighter) Be star/X-ray binaries using simultaneous optical/infrared/X-ray data will enable more detailed modelling of such systems to be performed. What is needed is long-term monitoring of several optical and infrared emission lines from the circumstellar disc throughout the whole X-ray outburst, and preferably over a complete orbital period.

\section{ACKNOWLEDGMENTS}

We thank the PATT for the allocation of observing time on the WHT and UKIRT, and are grateful for the support of service observing programmes on these telescopes and also the JKT. The WHT and JKT are operated by the Royal Greenwich Observatory at the Spanish Observatorio del Roque de los Muchachos of the Instituto de Astrofísica de Canarias, and the UKIRT is operated by the Royal Observatory, Edinburgh. Thanks to Chris Benn, Neil O'Mahoney and Roberto Terlevich at the WHT; Colin Aspin, Joel Aycock, John Davies, Tom Geballe, Dolores Walther and Thor Wold at UKIRT; and Skip Staples and Jean Mueller at Palomar for assistance during the observations and for obtaining service data. We also thank Derek Jones at the RGO for obtaining JKT service data; Marshall Cohen and Angela Putney for helpful information about operating the Palomar spectrograph; and Lars Bildsten for comments on drafts of this paper. $\mathrm{CE}$ acknowledges the support of a PPARC studentship. The data reduction was mainly carried out using the Southampton University Starlink node, which is funded by the PPARC.

\section{REFERENCES}

Apparao K.M.V., 1991, ApJ, 376, 256

Aspin C., Rayner J.T., McLean I.S., Hayashi S.S., 1990, MNRAS, 246, 565

Aspin C., Emerson J.P., Currie M.J., 1991, Starlink User Note, 41.3, R.A.L.

Bjorkmann J.E., Cassinelli J.P., 1993, ApJ, 409, 429

Clegg R.E.S., Carter D., Charles P., Dick J.S.B., Jenkins C.R., King D.L., Laing R.A., 1992, Isaac Newton Group, La Palma, User Manual No. XXII

Coe M.J., Longmore A., Payne B.J., Hanson C.G., 1988, MNRAS, 232,865

Coe M.J. et al., 1994, A\&A, in press

Fishman G.J. et al., 1989, in Johnson W.N., ed., Proceedings of Gamma Ray Observatory Science Workshop. NASA/GSFC, Greenbelt, p. 2

Gnedin Y.N., Pavlov G.G., Krisman M., Apparao K.M.V., Chitre S.M., 1981, Ap\&SS, 76, 105

Janot-Pacheco E., Motch C., Pakull M.W., 1988, A\&A, 202, 81

Jones J.J., Gehrz R.D., 1990, ApJ, 100, 274

Kirk J.G., Stoneham R.J., 1982, MNRAS, 201, 1183

Landolt A.U., 1983, AJ, 88, 439

Meyerdierks H., 1992, Starlink User Note, 86.8, R.A.L.

Motch C., Janot-Pacheco E., 1987, A\&A, 182, L55

Nagase F., 1989, PASJ, 41, 1

Oke J.B., Gunn J.E., 1982, PASP, 94, 586

Parmar A.N., White N.E., Stella L., Izzo C., Ferri P., 1989a, ApJ, 338, 359

Parmar A.N., White N.E., Stella L., 1989b, ApJ, 338, 373

Poeckert R., Bastien P., Landstreet J.D., 1979, ApJ, 84, 812

Prince T.A., Bildsten L., Chakrabarty D., Wilson R.B., Finger M.H., 1994, in Holt S.S., Day C.S., eds, AIP Conf. Proc. 308, The Evolution of X-ray Binaries. AIP, New York, p.235

Reynolds A.P., Parmar A.N., White N.E., 1993, ApJ, 414, 302

Rieke G.H., Lebofsky M.J., 1985, ApJ, 288, 618

Serkowski K., Mathewson D.S., Ford V.L., 1975, ApJ, 196, 261

Shortridge K., 1991, Starlink Miscellaneous User Document, 13, R.A.L.

Slettebak A., 1979, Space Sci. Rev., 23, 541

Slettebak A., Collins G.W., II, Truax R., 1992, ApJS, 81, 335

Stollberg M.T. et al., 1993, in Friedlander M., Gehrels N., Macomb D.J., eds, AIP Conf. Proc. 280, The Compton Gamma-Ray Observatory. AIP, New York, p.371

Stollberg M.T. et al., 1994, in Holt S.S., Day C.S., eds, AIP Conf. Proc. 308, The Evolution of X-ray Binaries. AIP, New York, p. 255

van den Heuvel E.P.J., Rappaport S., 1987, in Slettebak A., Snow T.P., eds, Proc. IAU Colloq. 92, Physics of Be stars. Cambridge Univ. Press, Cambridge, p.361 
$\underset{\infty}{\substack{c \\ \infty}} 992$ A.J. Norton et al.

Waters L.B.F.M., 1989, in Hunt I., Battrick B., eds, Proc. 23̈rd ESLAB Symposium ESA SP-296, X-ray Binaries. ESTEC, The Netherlands, p. 25

Waters L.B.F.M., Taylor A.R., van den Heuvel E.P.J., Habets G.M.H.J., Persi P., 1988, A\&A, 198, 200

White N.E., Swank J.H., Holt S.S., 1983, ApJ, 270, 711

Whittet D.C.B., van Breda I.G., 1978, A\&A, 66, 57
Wilking B.A., Lebofsky M.J., Martin P.G., Rieke G.H., Kemp J.C., 1980, ApJ, 235, 905

Zombeck M.V., 1990, Handbook of Space Astronomy and Astrophysics. Cambridge Univ. Press, Cambridge

This paper has been produced using the Royal Astronomical Society/Blackwell Science $\mathrm{LT}_{\mathrm{E}} \mathrm{X}$ style file. 